\title{
Trasplante alogénico de precursores hematopoyéticos en pacientes con síndrome de W iskott-Aldrich
}

\author{
María Angélica W ietstruck $P^{1}$, Pamela Zúñiga $C^{1}$, \\ Eduardo Talesnik $\mathbf{G}^{2}$, Cecilia Méndez $R^{2}$, \\ Francisco Barriga $\mathrm{C}^{1}$. \\ Hematopoietic stem cell \\ transplantation for patients \\ with Wiskott-Aldrich syndrome
}

\begin{abstract}
Background: Wiskott-Aldrich syndrome (WAS) is an X linked congenital disease that presents as eczema, thrombocytopenia and immune deficiency. The only curative procedure for this illness is hematopoietic stem cell transplant (HSCT), preferably from a healthy HLA identical sibling donor. Cord blood is becoming an excellent alternative as stem cell source from unrelated donors. Aim: To report our experience with HSCT in children with WAS. Patients and methods: Six boys with WAS diagnosed at 1 to 6 months of age were transplanted at our institution. All of them developed eczema and thrombocytopenia. Two had episodes of severe bleeding and three had repetitive infections (two with recurrent pulmonary infections and one a recurrent otitis). Three patients had a positive family history. Two received HSCT from sibling donors and four from unrelated cord blood donors at 7 months to 4 years of age. Results: All 6 patients had full hematopoietic engraftment after transplantation. Three had mild chronic graft-versus- host disease which responded to immune suppressive therapy. One patient died of cytomegalovirus related pneumonia 111 days after grafting. The other 5 patients are alive and healthy 11 to 104 months after transplantation. Conclusions: HSCT is an effective treatment for patients with WAS. The procedure should be done as soon as diagnosis is confirmed and before life threatening infections occur (Rev Méd Chile 2007; 135: 917-23).
\end{abstract}

(Key-w ords: Hematopoietic stem cells; Transplantation, homologous)

Recibido el 3 de enero, 2006. Aceptado el 29 de septiembre, 2006.

${ }^{1}$ Sección de Hematología-Oncología e ${ }^{2}$ Inmunología, Departamento de Pediatría, Escuela de Medicina, Pontificia Universidad Católica de Chile. Santiago de Chile.

Correspondencia a: Dra. María Angélica Wietstruck P. Departamento de Pediatría, Escuela de Medicina, Pontificia Universidad Católica de Chile. Lira 85, 5 piso. Santiago,

Chile. Fax: 638-4307. E mail: mwietstr@med.puc.cl 
$\mathrm{E}^{1}$ síndrome de Wiskott-Aldrich (SWA) es un desorden ligado al cromosoma $\mathrm{X}$, con una incidencia en la población de 4 por millón de hombres nacidos vivos ${ }^{1,2}$. La enfermedad resulta de una mutación que afecta al gen WAS localizado en el brazo corto del cromosoma X, identificado y secuenciado en 1994. El gen afectado codifica una proteína WASp, que interviene en la transmisión de señales desde los receptores de superficie al citoesqueleto, lo cual se traduce en defectos de la motilidad de las células inmunes, defectos de fagocitosis, alteraciones en la activación celular y alteraciones en la producción de plaquetas $^{3-5}$. Las manifestaciones clínicas que derivan de estos trastornos son: sangrado por trombocitopenia, infecciones recurrentes por defectos de la función inmune (tanto de células B como T), eczema y manifestaciones atópicas, y aparición de fenómenos autoinmunes ${ }^{1,6}$.

El tratamiento de esta enfermedad ha sido de soporte para prevenir hemorragias e infecciones. Sin embargo, en las formas más graves del síndrome, la mortalidad es alta y estos pacientes fallecen precozmente a causa de infecciones oportunistas o hemorragias. El trasplante alogénico de precursores hematopoyéticos (TAPH) se ha desarrollado en los últimos 25 años como tratamiento definitivo para algunos tipos de inmunodeficiencias severas, entre ellas el SWA ${ }^{8,14}$. Este procedimiento permite la corrección permanente del defecto inmune y hematológico. Sin embargo, la mayoría de los pacientes no cuentan con un donante familiar compatible, y es por esto que la búsqueda de fuentes alternativas de precursores hematopoyéticos, como la sangre de cordón umbilical, ha sido necesaria para el manejo de estos pacientes.

El objetivo del presente trabajo es presentar las características clínicas y evolución de 6 niños con SWA tratados con TAPH en Chile.

\section{PACIENTES Y MÉTODOS}

Desde 1996 hasta 2004 se recibieron 6 niños con SWA en la Unidad de Inmunología y Hematología Pediátrica de la Universidad Católica de Chile, tres de los cuales fueron diagnosticados en nuestra institución y los otros tres referidos al programa de trasplante. Todos los pacientes tenían trombo- citopenia y eczema; 4 presentaban infecciones recurrentes (en 3 eran predominantemente respiratorias, el otro paciente había presentado episodios de otitis), uno de ellos tuvo además una toxoplasmosis. La gravedad y frecuencia de las infecciones fueron directamente proporcionales a la edad de los pacientes, generalmente apareciendo después del primer año (dos pacientes). En 5 pacientes se realizó estudio de función inmune y todos éstos tenían algún tipo de alteración, desde hipogammaglobulinemia a disminución cuantitativa de linfocitos T. Dos pacientes tenían sangrados mucosos importantes (Tabla 1). Existe un score clínico internacional que define la gravedad de los síntomas, dando un puntaje de 1 a 5 basado en la intensidad de las manifestaciones clínicas arriba descritas $^{7}$, todos nuestros pacientes tenían puntaje de 3 ó 4.

En 2 pacientes el diagnóstico se confirmó por historia familiar fuertemente positiva (pacientes 1 y 2). Sólo en los pacientes 4,5 y 6 se pudo realizar el estudio de mutaciones. En el paciente 3, el diagnóstico fue clínico. Todos los pacientes recibieron terapia de soporte de acuerdo a su presentación clínica: los dos niños con hemorragias frecuentes recibieron transfusiones múltiples de plaquetas y glóbulos rojos. Tres pacientes recibieron terapia de reemplazo con inmunoglobulina endovenosa. Todos los que presentaron infecciones recibieron terapia antibiótica adecuada al cuadro clínico. El paciente que desarrolló toxoplasmosis fue tratado con eritromicina por 21 días previos al trasplante.

Sólo 2 de los 6 pacientes tenían hermanos histocompatibles. En los otros 4 se realizó búsqueda de unidades compatibles de sangre de cordón umbilical (SCU) en el Banco de Sangre de Nueva York, encontrándose en todos los casos al menos una.

Los 6 pacientes fueron tratados con TAPH, entre los 7 meses y los 4 años de vida. En todos, el condicionamiento se realizó con busulfán $40 \mathrm{mg} / \mathrm{m}^{2}$ vo cada $6 \mathrm{~h}$ por 16 dosis los días $-10,-9,-8,-7, \mathrm{y}$ ciclofosfamida $50 \mathrm{mg} / \mathrm{kg} /$ día los días $-6,-5,-4,-3$; los pacientes trasplantados con SCU recibieron, además, linfoglobulina $30 \mathrm{mg} / \mathrm{kg} /$ día los días -3, 2 , -1. Uno de éstos (№ 4) recibió, además, tiotepa como parte del condicionamiento (Tabla 2).

Los pacientes con donante hermano (2 y 3 ) recibieron dosis de células nucleadas de 7,7 y 
Tabla 1. C aracterísticas de los pacientes

\begin{tabular}{|c|c|c|c|c|c|c|c|}
\hline Paciente & $\begin{array}{c}\text { Edad al } \\
\text { diagnóstico } \\
\text { (meses) }\end{array}$ & $\begin{array}{l}\text { Familiares } \\
\text { fallecidos }\end{array}$ & $\begin{array}{l}\text { Hemo- } \\
\text { rragia }\end{array}$ & $\begin{array}{l}\text { Plaquetas } \\
1000 / \mathrm{mm}^{3}\end{array}$ & Eczema & Inmunidad & Infección \\
\hline 1 & 3 & $\begin{array}{l}11 \text { (tres } \\
\text { generaciones) }\end{array}$ & No & $40-70$ & + & $\downarrow$ IgA-M-G & Celulitis \\
\hline 2 & 2 & Tío materno & Rectal & $30-40$ & + & $\downarrow \operatorname{Linf} \mathrm{T}$ & No \\
\hline 3 & 6 & No & No & 20 & + & NR & $\begin{array}{l}\text { Neumonía, } \\
\text { toxoplasmosis }\end{array}$ \\
\hline 4 & 2 & No & No & 30 & + & $\downarrow \mathrm{IgM}, \mathrm{CD} 4$ & Neumonía \\
\hline 5 & 1 & Tío materno & Rectal & $10-90$ & + & $\begin{array}{c}\downarrow \mathrm{CD} 3, \mathrm{CD} 4 \\
\mathrm{y} \operatorname{Linf} \mathrm{B}\end{array}$ & No \\
\hline 6 & 1 & No & No & $20-60$ & + & $\downarrow \mathrm{CD} 8$ y CD19 & Otitis \\
\hline
\end{tabular}

NR: no realizado.

Tabla 2. C aracterísticas de los pacientes en relación al TAPH

\begin{tabular}{|c|c|c|c|c|}
\hline Paciente & Edad al TAPH & $\begin{array}{c}\text { Fuente } \\
\text { precursores }\end{array}$ & Condicionamiento & $\begin{array}{c}\text { Dosis celular* } \\
\left(10^{7} \mathrm{cel} / \mathrm{kg}\right)\end{array}$ \\
\hline 1 & 15 meses & SCU DNR & Bu-Cy-ATG & 8,6 \\
\hline 2 & 7 meses & $\begin{array}{l}\text { Hermana } \\
\text { HLA ID }\end{array}$ & Bu-Cy-ATG & 7,7 \\
\hline 3 & 48 meses & $\begin{array}{l}\text { Hermana } \\
\text { HLA ID }\end{array}$ & Bu-Cy & 2,1 \\
\hline 4 & 25 meses & SCU DNR & Bu-Сy-TTP-ATG & 8,4 \\
\hline 5 & 12 meses & SCU DNR & Bu-Cy-ATG & 2,6 \\
\hline 6 & 19 meses & SCU DNR & Bu-Cy-ATG & 2,7 \\
\hline
\end{tabular}

SCU DNR: Sangre de cordón umbilical de donante no relacionado. HLA id: HLA idéntico. Bu: Busulfan. Cy: ciclofosfamida. TTP: Tiotepa. ATG: Linfoglobulina.

*Dosis de células nucleadas infundidas, por kilo de peso del paciente.

2,1 x $10^{8}$ células por kilo, respectivamente. La recolección se hizo en pabellón, por múltiples aspiraciones de médula ósea. Los pacientes trasplantados con sangre de cordón umbilical de donante no relacionado recibieron entre 2,1 y 8,6 x $10^{7}$ células nucleadas $\mathrm{x}$ kilo de peso del paciente. La dosis de CD34 por kg del paciente la conocimos sólo en 3 de los 6 pacientes (ya que antes del año 1999 no contábamos con este examen), todos ellos recibieron SCU y varió entre 0,13 a 1,29 x $10^{6}$ CD34 por $\mathrm{kg}$. Las unidades de SCU escogidas eran compatibles en 4/6 ó 5/6 antígenos HLA con estudio de DRB1 de alta resolución, y se recibieron congeladas desde el New York Blood Center (Estados Unidos de Norteamérica).

La prevención de enfermedad de injerto contra huésped se hizo con ciclosporina $3 \mathrm{mg} / \mathrm{kg}$ cada 12 $\mathrm{h}$ EV desde el día -2 y metotrexato $15 \mathrm{mg} / \mathrm{m}^{2}$ (día 
$+1,+3,+6,+11)$ en receptores de médula ósea de donante hermano, y ciclosporina más metilprednisolona en receptores de sangre de cordón umbilical, $2 \mathrm{mg} / \mathrm{kg}$ hasta el día +20 , disminuyendo progresivamente $10 \%$ en forma semanal. La ciclosporina se mantuvo durante 6 meses, prolongándose hasta por 24 meses en caso de que se hiciera el diagnóstico clínico de enfermedad de injerto contra huésped.

Todos los pacientes fueron hospitalizados en piezas de aislamiento y recibieron tratamiento profiláctico con aciclovir $5 \mathrm{mg} / \mathrm{kg}$ cada $8 \mathrm{~h}$, a excepción del último paciente trasplantado con SCU, que recibió ganciclovir durante el condicionamiento por tener IgG para citomegalovirus (CMV) positiva, fluconazol $5 \mathrm{mg} / \mathrm{kg}$ día, y gammaglobulina $500 \mathrm{mg} / \mathrm{kg}$ cada semana desde la etapa de condicionamiento hasta la recuperación hematológica. Después del prendimiento continuaron con fluconazol, aciclovir y cotrimoxazol por un período de 6 meses o mientras durara la inmunosupresión.

\section{RESULTADOS}

La evolución clínica de nuestros pacientes se muestra en la Tabla 3. En todos hubo prendimiento y reconstitución hematológica: de glóbulos blancos
(RAN $>500 \times \mathrm{mm}^{3}$ durante 3 días consecutivos) entre el día +9 y +23 , y de plaquetas (recuento mayor de $20.000 \mathrm{x} \mathrm{mm}^{3}$ sin mediar transfusiones en los 7 días previos) entre el día +20 y +75 .

Tres pacientes desarrollaron enfermedad de injerto contra huésped (EICH) crónica localizada en la piel (el paciente № 6, además, presentó afectación gastrointestinal leve que consistió en diarrea de tres semanas de duración, a 7 meses del trasplante, sin agente infeccioso, que respondió a tratamiento esteroidal). Todos se trataron con ciclosporina o tacrolimus y corticoides. Uno de ellos, receptor de médula de hermana compatible, evolucionó a esclerodermia de predominio en pliegues, que se resolvió después de seis meses de inmunosupresión. Los tres niños actualmente están bien y sólo uno se mantiene con tratamiento inmunosupresor a 11 meses postrasplante.

Cuatro pacientes tenían serología positiva para CMV previo al trasplante y tres tuvieron reactivación de la infección posteriormente. Uno recibió médula ósea de un hermano y los otros 3 sangre de cordón umbilical. El primero presentó laringitis, la cual apareció en el día +18 postrasplante, con evolución inicial a obstrucción de la vía aérea superior que requirió intubación endotraqueal. Tuvo una buena respuesta a tratamiento con ganciclovir más inmunoglobulina endovenosa sin reactivaciones posteriores. De los tres pacientes

Tabla 3. Evolución postrasplante

\begin{tabular}{|ccclll|}
\hline Paciente & $\begin{array}{c}\text { RAN }>500 \\
\text { (día) }\end{array}$ & $\begin{array}{c}\text { Plq }>20.000 \\
\text { (día) }\end{array}$ & Infecciones & EICH & $\begin{array}{l}\text { Seguimiento (condición, } \\
\text { meses post TMO) }\end{array}$ \\
\hline 1 & 11 & 43 & $\begin{array}{l}\text { No } \\
\text { Bacteremia }\end{array}$ & $\begin{array}{l}\text { Crónica limitada } \\
\text { Crónica } \\
\text { generalizada } \\
\text { estafilococo } \\
\text { Bacteremia } \\
\text { Laringitis CMV }\end{array}$ & $\begin{array}{l}\text { Sano } 104 \text { meses } \\
\text { Sano } 101 \text { meses }\end{array}$ \\
3 & 9 & 75 & Sano 74 meses \\
4 & 12 & 20 & $\begin{array}{l}\text { Neumonía CMV } \\
\text { Reactivación CMV }\end{array}$ & $\begin{array}{l}\text { No } \\
\text { No } \\
\text { Crónica limitada }\end{array}$ & $\begin{array}{l}\text { Fallecido } 111 \text { días } \\
\text { Sano } 54 \text { meses } \\
\text { Sano 11 meses, en tto } \\
\text { inmunosupresor por } \\
\text { EICH }\end{array}$ \\
\hline
\end{tabular}

RAN: prendimiento de glóbulos blancos (recuento absoluto de neutrófilos $>500 / \mathrm{mm}^{3}$ ). Plq: prendimiento de plaquetas ( $>20.000 / \mathrm{mm}^{3}$ sin transfusión por 7 días). CMV: citomegalovirus. EICH: enfermedad de injerto contra huésped. CD: Clostridium difficile. ITU: infección del tracto urinario. 
CMV positivos que recibieron trasplante de SCU, dos presentaron reactivación de la infección; uno desarrolló una neumonitis grave falleciendo por esta causa en el día +111 , a pesar de tratamiento antiviral con ganciclovir, inmunoglobulina endovenosa y foscarnet; el otro paciente trasplantado con SCU y con reactivación de CMV tuvo persistencia de antigenemia positiva fluctuante por 6 meses, recibiendo tratamiento con ganciclovir endovenoso durante todo este período hasta negativizar la antigenemia. El tercer paciente con serología positiva recibió ganciclovir profiláctico durante el condicionamiento, manteniendo antigenemia negativa desde el trasplante a pesar de estar con tratamiento inmunosupresor por EICH crónica.

Sobreviven 5 niños postrasplante, con un rango de seguimiento entre 11 y 104 meses. Todos están bien, mantienen recuentos de plaquetas normales, sin eczema ni infecciones oportunistas. Cuatro han sido estudiados, demostrándose cifras de linfocitos (B, T y NK) e inmunoglobulinas en rangos normales.

\section{DisCUSIÓN}

El SWA es una enfermedad poco común y potencialmente fatal. Nuestra serie es similar a la publicada por otros grupos en relación al número de casos que pudieron acceder al TAPH. Hasta la década 1970-79, el único tratamiento de estos pacientes era de soporte y el promedio de vida de los mismos no pasaba de los 15 años. Las causas de fallecimiento de estos niños son infecciones (44\%), hemorragias (23\%) y enfermedades malignas asociadas $(26 \%)^{6}$. En 1968 se realizó con éxito el primer TAPH de médula ósea de un donante hermano compatible en un niño de 2 años portador de esta enfermedad ${ }^{8}$; desde entonces, este procedimiento se ha venido desarrollando como único tratamiento con potencial curativo en pacientes con SWA ${ }^{9,10}$. Sin embargo, esto sólo beneficia a no más de $20 \%$ de los pacientes, debido a la ausencia de donantes hermanos compatibles en las familias afectadas. Por ello se ha recurrido a donantes familiares no hermanos y donantes no relacionados obtenidos a través de registros de voluntarios. Estas modalidades de TAPH usualmente requieren de la manipulación del injerto para evitar el no prendimiento o la enfermedad de injerto versus huésped. Esto se realiza mediante depleción de linfocitos $\mathrm{T} 0$ enriquecimiento de células precursoras CD3410,11. Recientemente se ha empleado sangre de cordón umbilical como fuente de precursores hematopoiéticos, estableciéndose bancos de SCU para trasplante en diversas ciudades del mundo ${ }^{12}$. La ventaja de ésta es su pronta disponibilidad, la baja incidencia de enfermedad de injerto versus huésped asociada y la posibilidad de hacer trasplantes con unidades que tienen hasta dos diferencias mayores en su tipo HLA con el paciente ${ }^{13}$. La sobrevida de pacientes con SWA que se trasplantan fluctúa desde $80 \%-90 \%$ para los que reciben médula ósea de hermanos idénticos, comparado con $71 \%$ de donantes no relacionados y $53 \%$ de donante familiar no hermano ${ }^{14}$. Aún no se han publicado series numerosas con resultados a largo plazo de trasplante de SCU en esta patología, si bien los reportes de casos aislados han demostrado resultados muy promisorios ${ }^{15,16}$. Debido a esto, algunos centros en el extranjero han adoptado la estrategia de iniciar búsquedas simultáneas de donantes voluntarios y unidades de SCU cuando se enfrentan a un paciente pediátrico que necesita un trasplante alogénico de precursores hematopoiéticos y no tiene un donante en la familia ${ }^{17}$.

Nuestra serie de 6 pacientes demuestra la factibilidad de realizar este procedimiento en nuestro país, usando donantes hermanos o unidades de sangre de cordón de donantes no relacionados. No existen publicaciones nacionales que describan la evolución de los pacientes sin trasplante, pero debiera ser similar a lo que ya hemos comentado en relación al tratamiento de soporte de estos niños. En la actualidad, el trasplante se propone como tratamiento de elección para este grupo de pacientes siempre que se cumplan los siguientes requisitos: 1 . Que el diagnóstico de la enfermedad sea cierto, preferentemente con demostración de la mutación en el gen WAS. Los pacientes con la tríada clínica clásica (trombocitopenia, eczema e infecciones) sin historia familiar debenían confirmarse mediante este método. También es posible demostrar por citometría de flujo la ausencia de la proteína codificada por este gen en linfocitos de sangre periférica ${ }^{18,19}$. La gravedad de los síntomas no determina qué pacientes deben ir a trasplante; si bien todos los pacientes 
con score clínico ${ }^{7}$ mayor de 3 tienen la forma clásica y sintomática de la enfermedad, aquellos con score 1 ó 2 podrían progresar en el tiempo, por lo que el trasplante estaría indicado en caso de tener un familiar compatible. 2. Que se identifique un donante histocompatible adecuado, de preferencia un hermano HLA idéntico. Como las probabilidades de estos pacientes de tener tal donante es muy reducida, se recurre a donantes no emparentados, entre los cuales los bancos de sangre de cordón umbilical están emergiendo como la fuente más adecuada de progenitores hematopoiéticos, especialmente para niños pequeños con enfermedades congénitas. 3. Que el procedimiento se lleve a cabo en una unidad de trasplante de médula pediátrica con experiencia, especialmente si se elige un donante no emparentado. 4. Que los riesgos de la enfermedad y del procedimiento sean explicados extensamente a los padres de los niños, incluyendo la posibilidad de una complicación letal del mismo (por infecciones).

El uso de donantes haploidénticos con depleción de células $\mathrm{T}$ se ha intentado en pacientes en los que no se cuenta con donante HLA idéntico; sin embargo, los problemas presentados en relación a prendimiento, enfermedad de injerto contra huésped, desórdenes linfoproliferativos postrasplante y sobrevida (comparándolos con otro tipo de donante) han restringido su indicación. Esto se ha intentado revertir aumentando las dosis de precursores en el trasplante ${ }^{11}$.

La sangre de cordón umbilical ha sido una buena alternativa en los casos de pacientes con inmunodeficiencias en los que no se dispone de un donante de médula ósea histocompatible. Rubinstein y cols ${ }^{20}$ reportaron hasta el año 1999 un total de 864 trasplantes con SCU de donantes no relacionados, unidades que fueron suplidas por el Banco de Sangre de New York para centros de trasplantes en todo el mundo; de estos pacientes,

\section{REFERENCIAS}

1. Ochs H. The Wiskott-Aldrich Syndrome. Semin Hematol 1998; 35: 332-45.

2. Thrasher A, Kinnon C. The Wiskott Aldrich Syndrome. Clin Exp Inmunol 2000; 120: 2-9.

3. Brickell P, Katz D, Trasher A. Review. WiskottAldrich Syndrome: Current Research Concepts.
209 tenían enfermedades genéticas (24\%), siendo 19 portadores de SWA (9\%); ellos encontraron que la dosis de células por kilo era el factor determinante para el prendimiento y que la sobrevida libre de enfermedad en pacientes con enfermedades genéticas era superior a la que se observó en niños con enfermedades malignas ( $48 \%$ vs $27 \%$ respectivamente, a 3 años postrasplante). Por otra parte, Gluckman y col ${ }^{21}$ analizaron los resultados de 291 trasplantes con SCU de donantes no relacionados realizados a través de Eurocord desde octubre de 1988 hasta enero de 2001; de éstos, 61 pacientes eran portadores de enfermedades genéticas (20,9\%); la sobrevida libre de eventos a 2 años fue de $51 \%$ en pacientes con errores congénitos, la probabilidad de prendimiento al día 60 fue alta (82\%) y la incidencia de EICH grado II-IV fue de $39 \%$. Ninguno de los dos trabajos especifica los resultados en esta enfermedad.

Nuestra serie de 6 pacientes confirma la relativa seguridad y eficacia del trasplante de células progenitoras hematopoiéticas para el tratamiento y corrección del SWA. El prendimiento tanto de glóbulos blancos como de plaquetas fue posible en todos ellos, aun a pesar de una dosis celular más baja en los que recibieron SCU; la EICH en nuestra serie fue más bien crónica y manejable; las infecciones representaron la complicación más frecuente, siendo la causa de muerte en uno de los pacientes a causa de neumonía grave por CMV.

En conclusión, el trasplante de células progenitoras hematopoiéticas debe ser considerado en todo paciente portador de SWA; el diagnóstico de la enfermedad deberá confirmarse con estudio de mutaciones; el uso de un donante no relacionado es factible en nuestro medio; los resultados han sido muy satisfactorios y deberá hacerse hincapié en las medidas que permitan prevenir complicaciones potencialmente fatales (como infecciones y EICH).

Br Journal Hematol 1998; 101: 603-8.

4. Kwan S, Hagemann T, Bradtke B, Biaese M, Rosen F. Identification of mutations in the Wiskott-Aldrich syndrome gene and characterization of a polymorphic dinucleotide repeat at DXS6940, adyacent to the disease gene. Proc Natl Acad Sci 1995; 92: 4706-10.

5. Thompson L, LaLLz M, Layton M. Unique and recurrent WAS gene mutations in Wiskott-Aldrich 
Syndrome and X-Linked Thrombocytopenia. Blood Cells, Mol and Dis 1999; 25: 218-26.

6. Suluvan K, Mulen C, Biaese M, Winkeistein J. A multiinstitutional survey of the Wiskott-Aldrich Syndrome. J Ped 1994; 125: 876-85.

7. Zhu Q, Zhang M, Blaese RM, Derry JM, Junker A. The Wiskott-Aldrich Syndrome and X-linked congenital thrombocytopenia are caused by mutations of the same gene. Blood 1995; 86: 3797-804.

8. Bortin M, Bach F, Van BekKum D, Good R, Van Rood J. Special Report. 25 th anniversary of the first successful allogeneic bone marrow transplants. Bone Marrow Transpl 1994; 14: 211-2.

9. Fischer A, Landais P, Friedrich B, Gerritsen F, Fasth F, Porta A et al. Bone Marrow Transplantation (BMT) in Europe for primary inmunodeficiencies other than severe combined immunodeficiency: a report from the European Group for BMT and the European Group for Inmunodeficiencies. Blood 1994; 83: 1149-54.

10. Brochstein J, Giшo A, Ruggiero M, Kernan N, EMANUEl D, LAVER J ET AL. Marrow transplantation from human leukocyte antigen-identical or haploidentical donors for correction of WiskottAldrich syndrome. J Ped 1991; 19: 907-12.

11. Lang P, Kungebiel T, Bader P, Greil J, Schumm M, SCHLEGEL PG ET AL. Transplantation of highly purified peripheral-blood CD34+ progenitor cells from related and unrelated donors in children with nonmalignant diseases. Bone Marrow Transplant 2004; 33: 25-32.

12. Rubinstein P, Adamson JW, Stevens C. The Placental/Umbilical Cord Blood Program of the New York Blood Center. A progress report. Ann N Y Acad Sci 1999; 872: 328-34.

13. Rubinstein P, Stevens CE. Placental blood for bone marrow replacement: the New York Blood Center's program and clinical results. Baillieres Best Pract Res Clin Haematol 2000; 13: 565-84.
14. Filipovich A, Stone J, Tomany S, Ireiand M, Kolman C, PeIZ C ET AL. Impact of donor type on outcome of bone marrow transplantation for WiskottAldrich syndrome: collaborative study of the International Bone Marrow Transplant Registry and the National Marrow Donor Program. Blood 2001; 97: 1598-610.

15. Kaneko M, Watanabe T, Watanabe H, Kimura $M$, SuzUYa H, Окамото Y ET al. Successful unrelated cord blood transplantation in an infant with Wiskott-Aldrich syndrome following recurent cytomegalovirus disease. Int J Hematol 2003; 78: 457-60.

16. KnutSen AP, Stefren M, Wassmer K, Wall DA. Umbilical cord blood transplantation in Wiskott Aldrich syndrome. J Pediatr 2003; 142: 519-23.

17. Dalie JH, Duval M, Moghrabi A, Wagner E, Vachon M, BARRETT S ET AL. Results of an unrelated transplant search strategy using partially HLAmismatched cord blood as an immediate alternative to HLA-matched bone marrow. Bone Marrow Transplant 2004; 33: 605-11.

18. Kanegane H, Nomura K, Miyanaki T, Sasahara $Y$, Kawai $\mathrm{S}$, Tsuchira $\mathrm{S}$ Eт AL. To the editor: X-linked thrombocytopenia identified by flow cytometric demonstration of defective Wiskott-Aldrich syndrome protein in lymphocytes. Blood 2000; 95: 1110-1.

19. Yamada M, Ohtsu M, Kobayashi I, Kawamura N, Kobayashi K, ARIGa T et al. Flow cytometric analysis of Wiskott-Aldrich syndrome (WAS) protein in lymphocytes from patients and their familial carriers. Blood 1999; 93: 756-58.

20. Rubinstein P, Carrier C, Scaradavou A, Kurtzberg J, adamson J, Miglaccio AR et al. Outcomes among 562 recipients of placental-blood transplants from unrelated donors. N Engl J Med 1998; 339: 1565-77.

21. Gluckman E, Rocha V, Chevret S. Results of unrelated umbilical cord blood hematopoietic stem cell transplantation. Rev Clin Exp Hematol 2001; 2: 87-99.

Agradecimientos

Los autores agradecen a los Dres. Pablo Rubinstein y Carmelita Carrier del New York Blood Center y a la Dra. Joanne Kurtzberg de la Universidad de Duke (Durham, NC) por todo el apoyo técnico y logístico para implementar nuestro programa de trasplante alogénico de sangre de condón y al Dr. Hans Ochs de la Universidad de Washington (Seattle, WA) quien detectó la mutación del gen WAS en el paciente 5. 